\title{
Cognitive Cycle in Mind Model CAM*
}

\author{
Zhongzhi Shi, Xiaofeng Wang, Jinpeng Yue \\ The Key Laboratory of Intelligent Information Processing, Institute of Computing Technology, \\ Chinese Academy of Sciences, Beijing, China \\ E-mail: shizz@ics.ict.ac.cn
}

Received October 2, 2011; revised October 10, 2011; accepted October 15, 2011

\begin{abstract}
Cognitive cycle is a basic procedure of mental activities in cognitive level. Human cognition consists of cascading cycles of recurring brain events. This paper presents a cognitive cycle for the mind model CAM (Consciousness and Memory). Each cognitive cycle perceives the current situation, through motivation phase with reference to ongoing goals, and then composes internal or external action streams to reach the goals in response. We use dynamic description logic which is an extended description logic with action to formalize descriptions and algorithms of cognitive cycle. Two important algorithms, including hierarchical goal and action composition, are proposed in the paper.
\end{abstract}

Keywords: Cognitive Cycle, Motivation, Action Composition, CAM

\section{Introduction}

Cognitive cycle is a basic procedure of mental activities in cognitive level. Human cognition consists of cascadeing cycles of recurring brain events. In problem solving, particular for production systems, solving cycles were proposed. In the early 1980 's, SOAR was developed to be a system that could support multiple problem solving methods for many different problems [1]. In the mid 1980's, Newell and many of his students began working on SOAR as a candidate of unified theories of cognition [2]. SOAR is a classic example of expert rule-based cognitive architecture designed to model general intelligence with a learning architecture that has been applied to domains ranging from rapid, immediate tasks such as typing and video game interaction to long stretches of problem solving behavior. SOAR has also served as the foundation for a detailed theory of sentence processing, which models both the rapid on-line effects of semantics and context, as well as subtle effects of syntactic structure on processing difficulty across several typologically distinct languages.

The adaptive control of thought-rational (ACT-R) model, developed mainly by Anderson [3], which is a symbolic cognitive architecture aiming to explain how the components of the mind work together to produce

${ }^{*}$ This paper is supported by Key projects of National Natural Science Foundation of China (No. 61035003, 60933004), National Natural Science Foundation of China (No. 61072085, 60970088, 60903141), National Basic Research Programme (2007CB311004). coherent cognition. Coordination of the ACT-R modules is achieved by a central production system shown in Figure 1 [4].

ACT-R is a hybrid cognitive architecture. Its symbolic structure is a production system; the subsymbolic structure is represented by a set of massively parallel processes that can be summarized by a number of mathematical equations. The subsymbolic equations control many of the symbolic processes. If several productions match the state of the buffers, a subsymbolic utility equation estimates the relative cost and benefit associ-

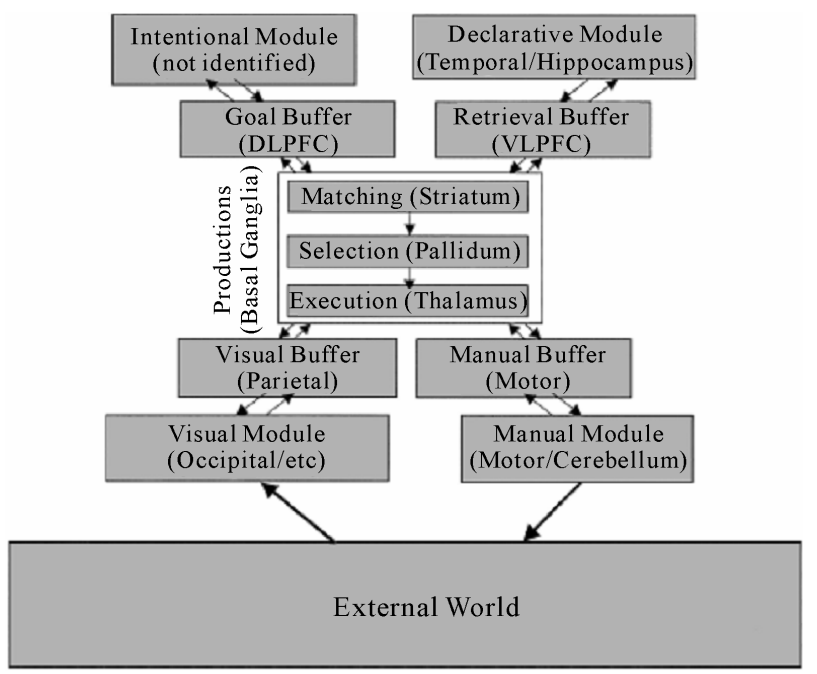

Figure 1. ACT-R architecture [4]. 
ated with each production and decides to select for execution the production with the highest utility.

In practice ACT-R seems to be used more as a programming framework for cognitive modeling than as an AI system. One can easily use ACT-R to program models of specific human mental behaviors, which may then be matched against psychological and neurobiological data [5]. Recently Anderson and his colleagues investigateACT-R under fMRI guide to execute algebra operations $[6,7]$.

An agent perceives the environment through the sensor and effect the environment with the actor to communicate with environment [8]. A human being, if we see it an agent, perceives environment with eyes, ear, nose, etc. It affects the environment using hands, legs etc. A robot agent usually uses some cameras for getting environment information and the motor is their actor. A software agent uses codes as their sensor and actor. In 1993, Shoham proposed agent-oriented programming in terms of BDI agent architectures [9]. The Belief-Desire-Intention (BDI) agent model is an event-driven execution model providing both reactive and proactive behavior. The BDI agent model is built on a simplified view of human intelligence. In it, agents have a view of the world (Beliefs), certain goals they wish to achieve (Desires), and they form Plans (Intentions) to act on these using their accumulated experience. Agents based on the BDI model are at a level of abstraction closer to normal human experience [10].

In AI terms, Beliefs represent knowledge of the world. However, in computational terms, Beliefs are just some way of representing the state of the world, be it as the value of a variable, a relational database, or symbolic expressions in predicate calculus. Beliefs are essential because the world is dynamic, and the system only has a local view of the world. Moreover, as the system is resource bounded, it is desirable to cache important information rather than recompute it from base perceptual data. Desires form another essential component of system state. Again, in computational terms, a Goal may simply be the value of a variable, a record structure, or a symbolic expression in some logic. The important point is that a Goal represents some desired end states. The underlying semantics for Goals, irrespective of how they are represented computationally, should reflect some logic of desire. In the AI literature, Intentions represent the third necessary component of system state. Computationally, Intentions may simply be a set of executing threads in a process that can be appropriately interrupted upon receiving feedback from the possibly changing world. The basic components of a system designed for a dynamic, uncertain world should include some representation of Beliefs, Desires, Intentions and Plans, or what has come to be called a BDI agent [11].

CLARION is a hybrid architecture that combines a symbolic component for reasoning on explicit knowledge' with a connectionist component for managing implicit knowledge. [12] Learning of implicit knowledge may be done via neural net, reinforcement learning, or other methods. The integration of symbolic and subsymbolic methods is powerful, but a great deal is still missing such as episodic knowledge and learning and creativity. Learning in the symbolic and subsymbolic portions is carried out separately rather than dynamically coupled, minimizing "cognitive synergy" effects. CLARION consists of a number of distinct subsystems, each of which contains a dual representational structure, including a "rules and chunks" symbolic knowledge store somewhat similar to ACT-R, and a neural net knowledge store embodying implicit knowledge. The CLARION architecture is shown in Figure 2. It contains main subsystems as follows:

1) An action-centered subsystem to control actions;

2) A non-action-centered subsystem to maintain general knowledge;

3) A motivational subsystem to provide underlying motivations for perception, action, and cognition;

4) A meta-cognitive subsystem to monitor, direct, and modify the operations of all the other subsystems.

Motivational dynamics is an essential part of human or animal behaviors. In CLARION Sun proposed a motivational subsystem shown in Figure 3 [13]. In this subsystem, the goal structure constitutes an explicit representation of motivations, and drives an implicit one. The mapping between the state of the world, for instance, stimuli as perceived by a cognitive agent, and the sensing of various perceived deficits, and the strengths of various drives can be implemented, in accordance with the aforespecified value ranges and relations, by back-propagation networks. The networks identify relevant features from raw sensory input. The output of such a network may be the strengths of drives.

The LIDA architecture developed by Stan Franklin and his colleagues is based on the concept of the cognitive cycle-a notion that is important to the brain [14, 15]. Each cognitive cycle the LIDA agent first makes sense of its current situation as best as it can. It then decides what portion of this situation is most in need of attention. Broadcasting this portion, the current contents of consciousness, enables the agent to finally choose an appropriate action and execute it.

Autonomous agents cope with their changing environment by their continuous, cyclic chores of "perceiveunderstand-act". LIDA's cognitive cycle is the cycle of refined cognitive processes that bring about the appropriate action for specific situation. As Franklin and Baars 


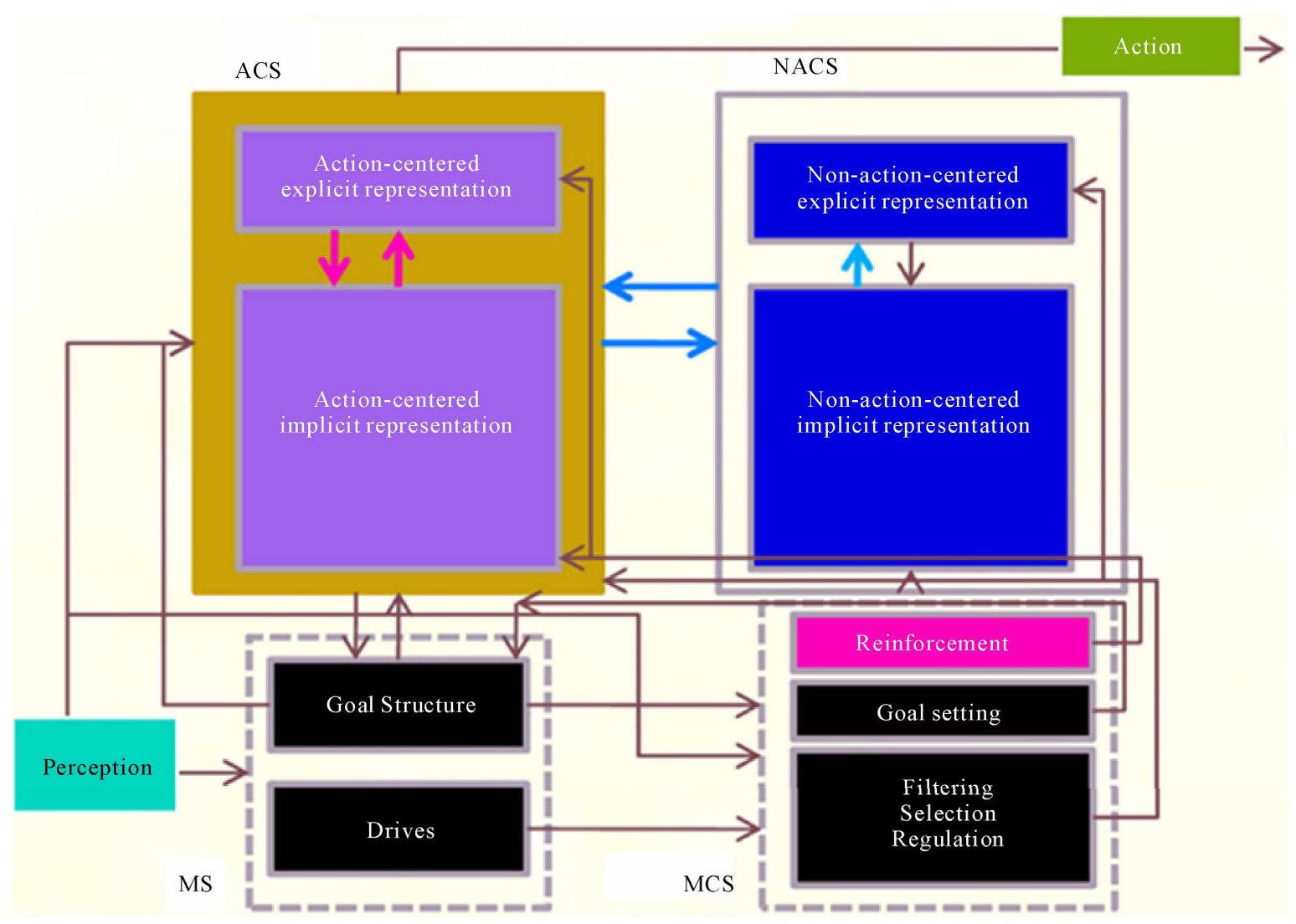

Figure 2. CLARION architecture [12].

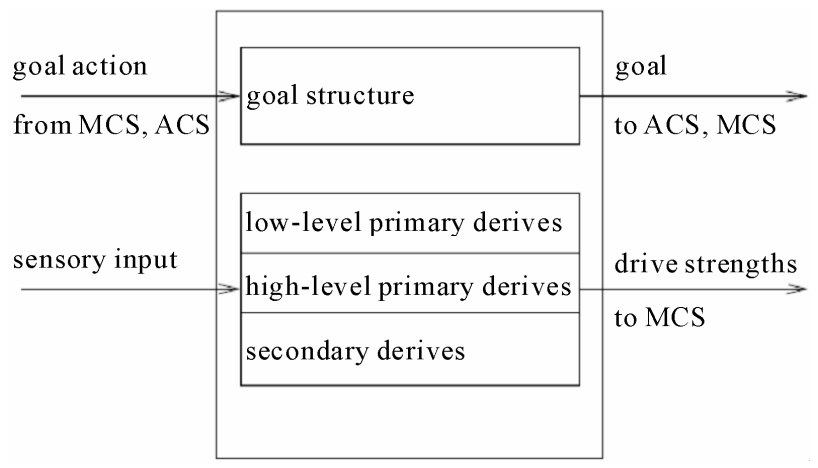

Figure 3. Structure of the motivational subsystem [13].

put it "A cognitive cycle can be thought of as a moment of cognition-a cognitive moment; higher-level cognitive processes are composed of many of these cognitive cycles, each a cognitive atom." This metaphor is to say that the steps in a cognitive cycle correspond to the various sub-atomic particles in an atom. Since the LIDA architecture is composed of several specialized mechanisms, a continual process that causes the functional interaction among the various components is essential. The cognitive cycle as such is an iterative, cyclical, continually active process that brings about the interplay among the various components of the architecture [16].
Cognitive cycle should reflect agent interaction with environment, which is sensory outside environment, through deliberation, and then effect environment. Cognitive cycle is a whole procedure from perception to behavior execution. In this paper we propose a cognitive cycle in mind model CAM which consists of three phases: perception, motivation and action composition. Dynamic description logic is used for formalizing descriptions and algorithms. Two important algorithms, including hierarchical goal and action composition, are proposed in the paper.

Next section will introduce mind model CAM. The CAM cognitive cycle will be discussed in Section 3. Finally, conclusion and perspective will be given.

\section{Mind Model CAM}

Mind could be defined as: “That which thinks, reasons, perceives, wills, and feels. The mind now appears in no way separate from the brain. In neuroscience, there is no duality between the mind and body. They are one.” in Medical Dictionary [17]. A mind model is intended to be an explanation of how some aspect of cognition is accomplished by a set of primitive computational processes. A model performs a specific cognitive task or class of 
tasks and produces behavior that constitutes a set of predictions that can be compared to data from human performance. Task domains that have received considerable attention include problem solving, language comprehension, memory tasks, and human-device interaction.

A new mind model called Consciousness and Memory (CAM) is proposed by Intelligence Science Laboratory of Institute of Computing Technology [18]. Figure 4 shows the architecture of CAM model which consists of three main parts, consciousness, memory and high level cognitive functions. The consciousness possesses a set of planning schemes which arrange the components of CAM to accomplish different cognitive tasks. The memory part contains three types of memory which are long term memory, short term memory and working memory. The high level cognitive function part includes event detection, action execution etc.

\subsection{Semantic Memory}

In semantic memory, that is, conceptual memory in Figure 4, we use ontology to specify a conceptualization of a domain in terms of concepts, attributes, and relations. The concepts provide model entities of interest in the domain. They are typically organized into a taxonomy tree where each node represents a concept and each concept is a specialization of its parent. Each concept in a taxonomy is associated with a set of instances. By the taxonomy's definition, the instances of a concept are also instances of an ancestor concept. Each concept is also associated with a set of attributes. An ontology also defines a set of relations among its concepts. Logic language Dynamic description logic (DDL) proposed by authors' Lab defines ontology terminologies and expressiveness [19].

An example of semantic memory to describe animal hierarchical category is given in Figure 5. We can describe it in DDL as follows:

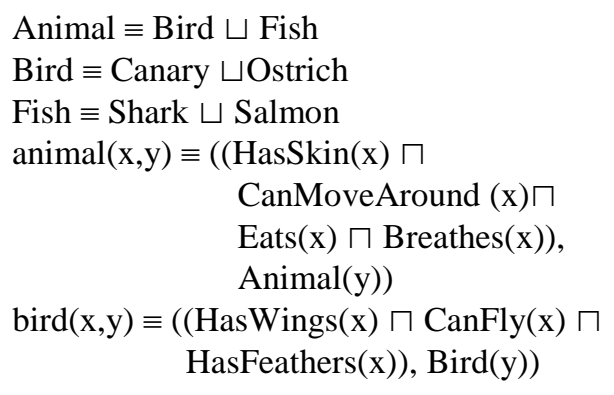

An example of semantic memory to describe animal hierarchical category is given in Figure 5. We can describe it in DDL as follows:

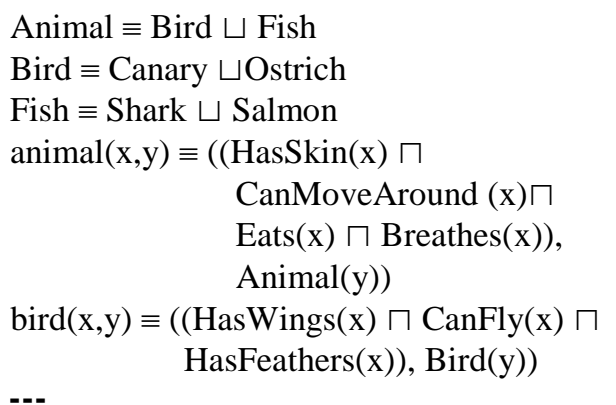

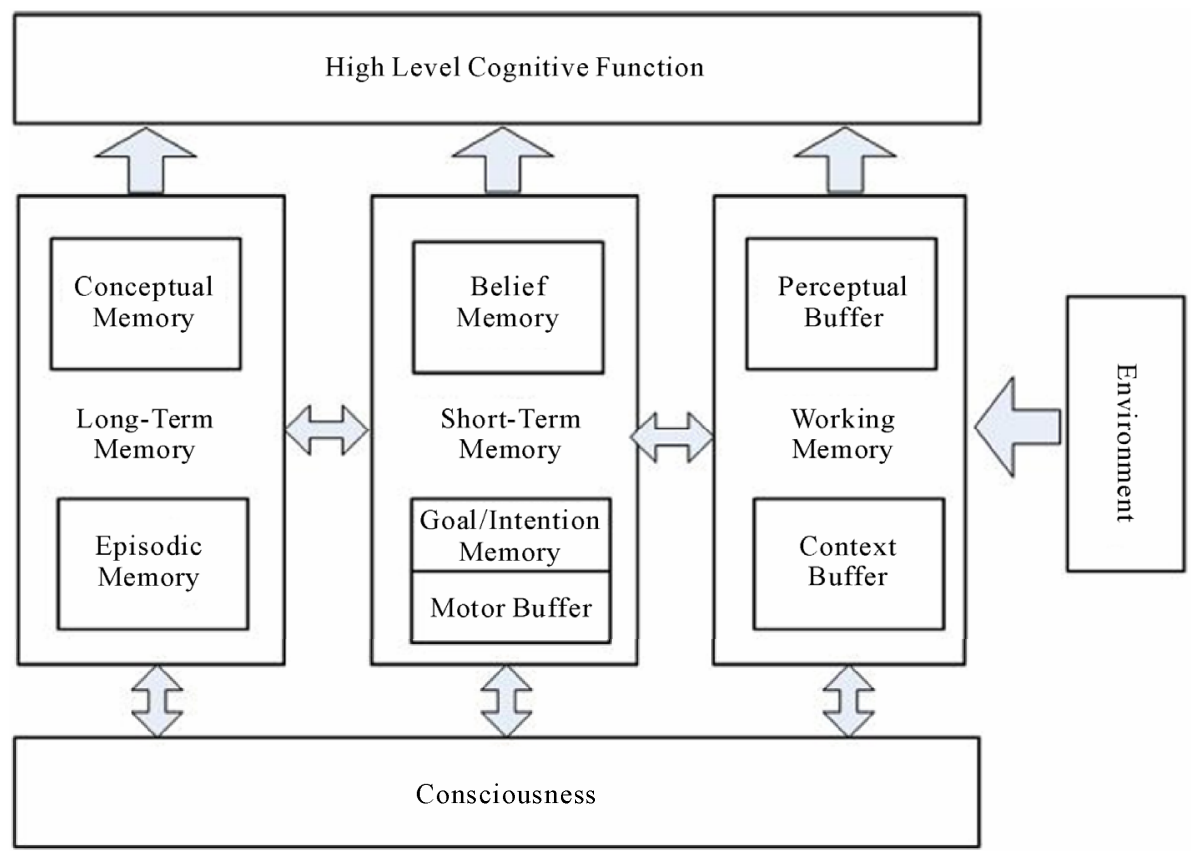

Figure 4. Architecture of CAM. 


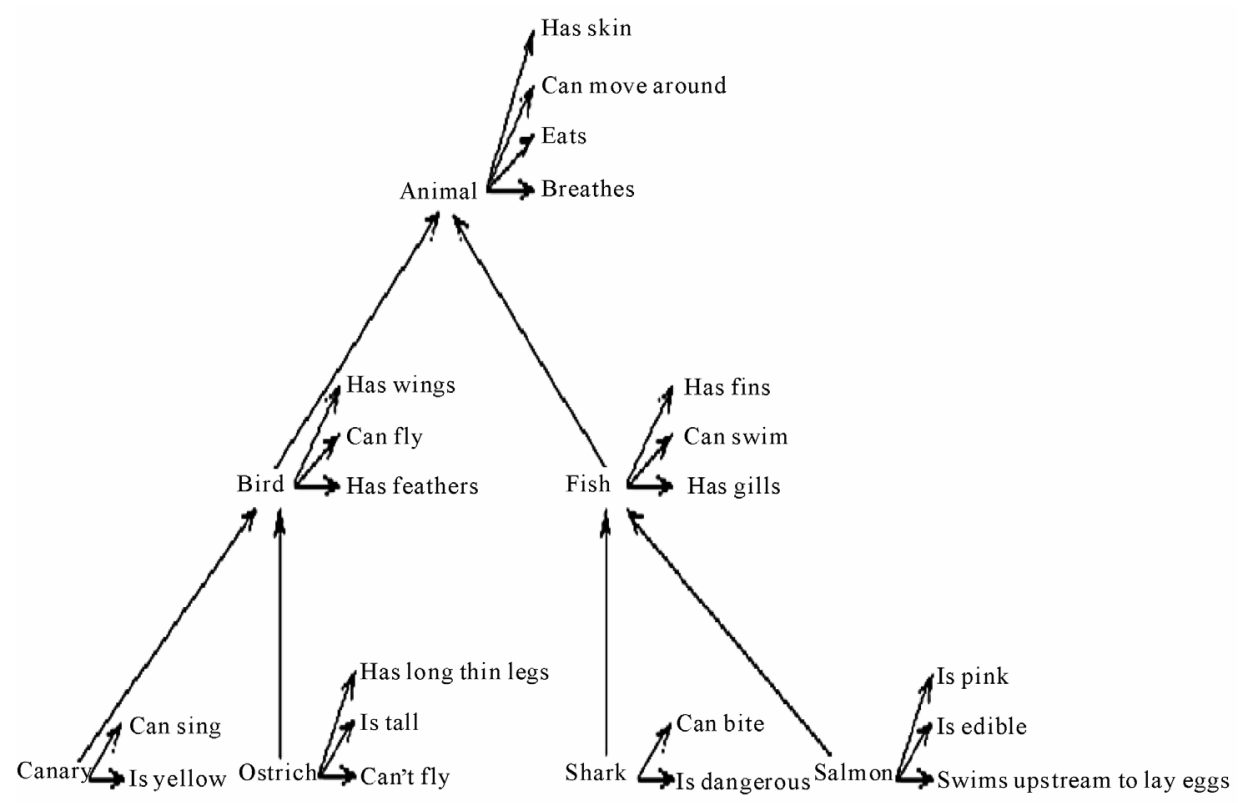

Figure 5. Semantic memory [20].

\subsection{Episodic Memory}

Episodic memory is a category of long-term memory that involves the recollection of specific events, situations and experiences [21]. Nuxoll and Laird demonstrated that an episodic memory can support an intelligent agent to own a multitude of cognitive capabilities [22].

In CAM the episode is an elementary unit that stores previous scene in episodic memory where an episode is divided into two levels: one is an abstract level in terms of logic, another is a primitive level shown in Figure 6. Among them, episode is represented in the form of logic symbol on the abstract level. The primitive level includes perception information correlated to abstract level of the described object.

In order to represent and organize perception of the episode effectively, we adopt DDL to describe episode in abstract level and ontology in primitive level. Object data graph (ODG) is used to describe episode. Figure 7 depicts an ODG structure of film Waterloo bridge where objects associate with other objects through URI in episode. Figure 7 shows us 3 objects: $\mathrm{M}_{2}, \mathrm{~W}_{2}$, and film Waterloo bridge. In addition, object $\mathrm{W}_{2}$ has worn a blue skirt. The film also associates with two main roles $\mathrm{M}_{1}$, $\mathrm{W}_{1}$ and among them $\mathrm{W}_{1}$ has worn a white coat.

In Soar, the retrieval of episode is modeled as a case-based reasoning problem which finds solutions to problems according previous experience [22]. We follow this idea and build a case based system to retrieve the episode according to the cues. To simplify the system, we restrict the cue to be transitional sequence like episode. Then, the retrieval of episode is modeled as prob-

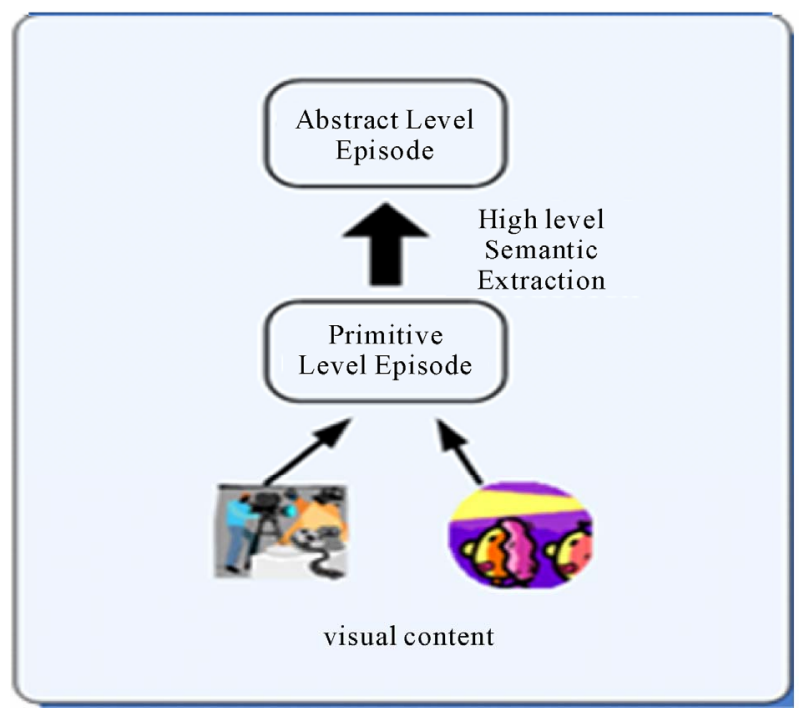

Figure 6. Example of episode.

lem of finding the episode that is most relevant to the cue. As the abstract level episode can represent the content of episode precisely, thus the matchmaking is only performed between cue and abstract level episode. In CAM, the transitional sequence is formally defined as possible world sequence and whether the episode implies cue can be inferred by the DDL based tableau algorithms [18].

\section{CAM Cognitive Cycle}

In mind model CAM we propose the cognitive cycle shown in Figure 8. The CAM cognitive cycle depicts as Perception-Motivation-Action Composition three phases. 


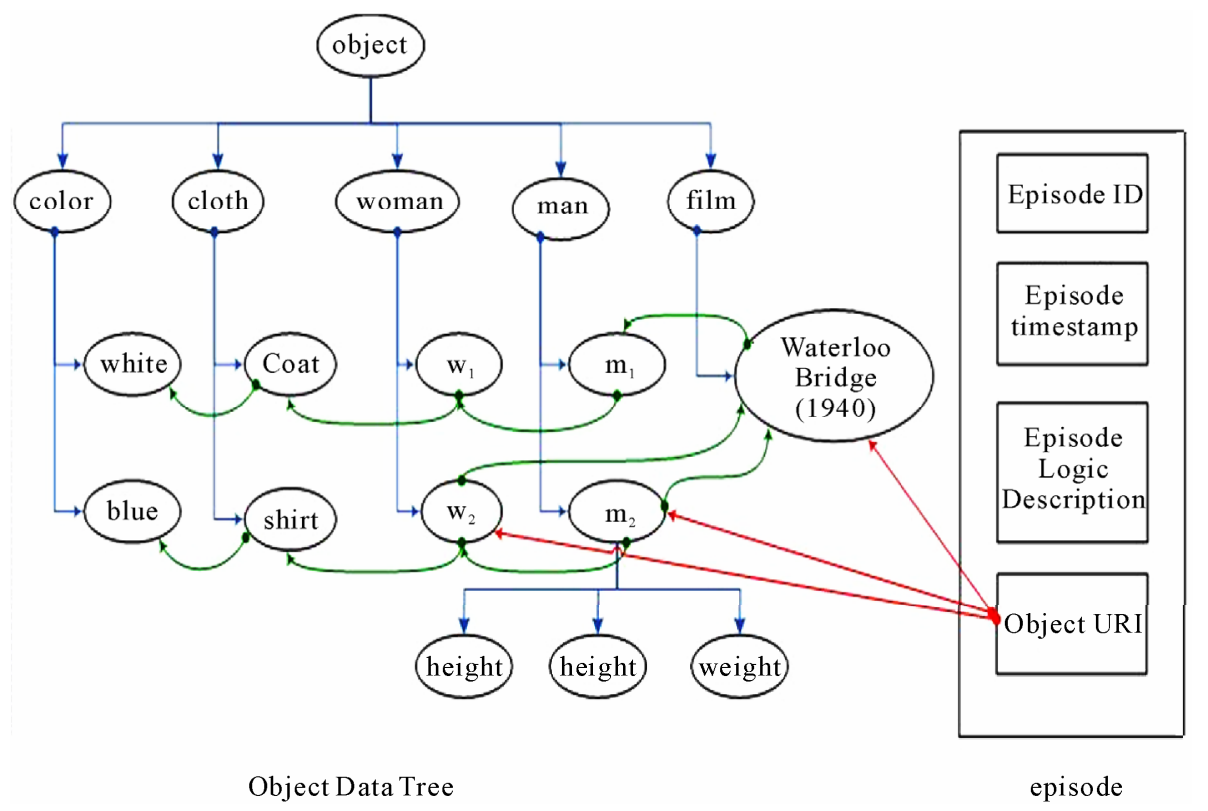

Figure 7. Object data graph.

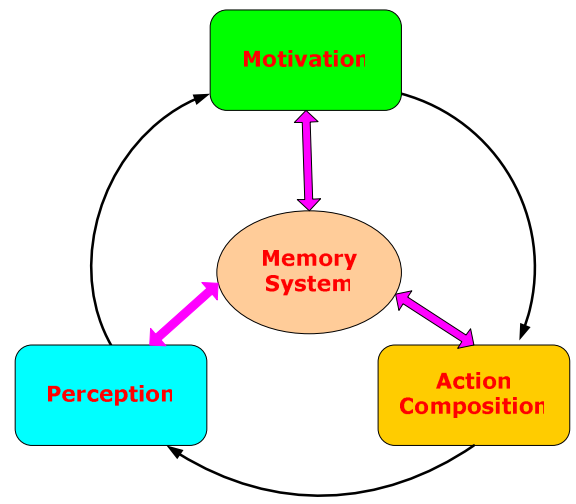

Figure 8. CAM cognitive cycle.

Perception phase is the process of attaining awareness of the environment by sensory input. Using the incoming percept and the residual contents of working memory, as cues, local associations are automatically retrieved from transient episodic memory and from declarative memory. Motivation phase focuses on learners' beliefs, expectations, and needs for order and understanding. According to the impact factors of motivation, such as proportional activation, opportunism, contiguity of action, persistence, interruption, combination of preference we construct a motivation subsystem. Action composition will compose a group of actions through action selection, planning to reach the end goal.

\subsection{Perception}

Perception phase is the process of attaining awareness or understanding of the environment by organizing and interpreting sensory information [23,24]. All perception involves signals in the nervous system, which in turn result from physical stimulation of the sense organs. Sensory stimuli, external or internal, are received and interpreted by perception producing the beginnings of meaning.

Awareness is the state or ability to perceive, to feel, or to be conscious of events, objects or sensory patterns. In this level of consciousness, sense data can be confirmed by an observer without necessarily implying understanding. More broadly, it is the state or quality of being aware of something. In biological psychology, awareness is defined as a human's or an animal's perception and cognitive reaction to a condition or event.

\subsection{Motivation}

Motivation is a process that starts with a physiological or psychological need that activates a behavior or a drive that is aimed at a goal. There are several motivation theories, such as Extrinsic motivation, Behaviorism, Humanistic views of motivation, Cognitive theories of motivation.

Physiologist Maslow developed the hierarchy of needs through his paper titled a Theory of Human Motivation in 1943 [25]. Since it introduced to the public, the Maslow's hierarchy of needs theory has been made a significant impact to the every life aspect in people's life. This theory can give people more spirit and motivation so they can manage their life very well [26]. The Maslow's hierarchy of needs is describing the reality of most people life experience accurately. The Maslow's 
hierarchy of needs theory is divided into five different levels of basic needs, including physiological needs in the lowest level, security needs in the second level, needs of love, affection and ownership in the next level, esteem needs in the fourth level, and the last is self-actualization needs in the top of hierarchy shown in Figure 9. Maslow actually was a humanistic psychologist who believed in the human potential that human can struggle to reach the success and look for the creativity in order to reach the highest wisdom and also the logic think. From above we can see that humanistic views of motivation focus on the learner as a whole person and examine the relationships among physical, emotional, intellectual, and aesthetic needs.

Bach has proposed a framework for an extensible motivational system of cognitive agents, based on research in psychology [27]. It draws on a finite set of pre-defined drives, which relate to needs of the system. Goals are established through reinforcement learning by interacting with an environment. Bach also points out that all behavior of Psi agents is directed towards a goal situation, which is characterized by a consumptive action satisfying one of the needs. Bach proposes hierarchy of agent needs with three levels shown in Figure 10. The lowest

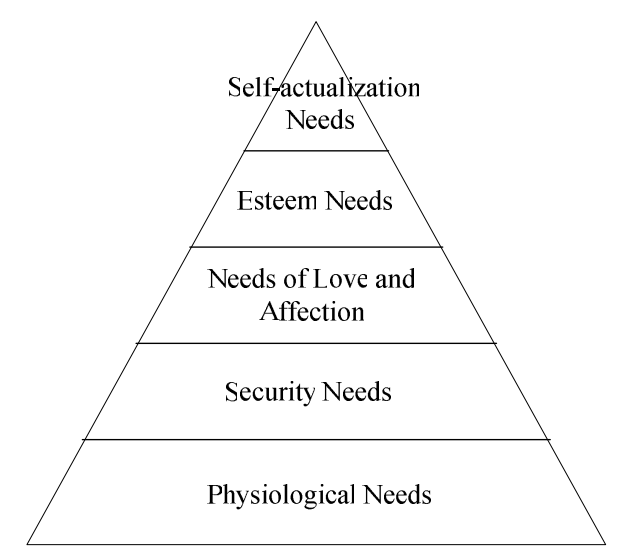

Figure 9. Hierarchy of needs.

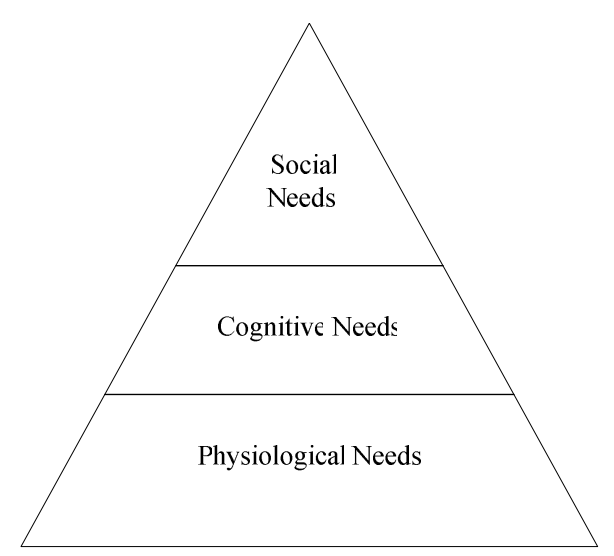

Figure 10. Hierarchy of agent needs. level is physiological needs, containing fuel and water, intactness. Second level is cognitive needs, containing certainty, competence, and aesthetics. The third level is social needs, containing affiliation and supplication signals.

Bach uses Psi theory to define a possible solution for a drive-based, poly-thematic motivational system. It can reflect physiological needs, also addresses the establishment of cognitive and social goals. Its straightforward integration of needs allows adapting it quickly to different environments and types of agents. They develop a version of the model which has been successfully evaluated against human performance in problem solving games [28].

In the motivation phase of the mind model CAM, an explicit goal may be set based on drives according to the needs. A goal list consists of a number of goals which can be described formally:

$$
G_{t}=\left\{G_{1}^{t}, G_{2}^{t}, \cdots, G_{n}^{t}\right\} \text { at time } t
$$

Definition 1: A hierarchical goal which is a directed acyclic graph (DAG) can be defined as a 3-tuples:

$$
\mathrm{DAG}=(P, E, \prec)
$$

where

$P$ : a set of nodes

$E$ : a set of edges which indicate the relation between connected nodes

$\prec$ : partial order

Should be satisfied following conditions:

1) A particular node TopGoal is contained in $P$, $\forall p \in P, \quad p \neq$ TopGoal , and $p \prec$ TopGoal

2) if $p_{1}, p_{2} \in P$, then $p_{1} \neq p_{2}$;

3) if $<p_{1}, p_{2}>\in E$, then $p_{2} \prec p_{1}$;

4) if $p \in P, \exists p_{1}, p_{2}, \cdots, p_{n} \in P$, and

$$
<p, p_{1}>\in E,<p_{1}, p_{2}>\in E,
$$

$$
<\mathrm{p}_{n-1}, p_{n}>\in E
$$

then $<p, p_{2}>\notin E, \cdots,<p, p_{n}>\notin E$.

Let a set of goals $G_{t}$ at time $t$ be

$G_{t}=\left\{G_{1}^{t}, G_{2}^{t}, \cdots, G_{n}^{t}\right\}$, where, $G_{i}^{t}, 1 \leq i \leq n$, let

$G_{i}^{t}=\left\{G_{i_{1}}^{t}, G_{i_{2}}^{t}, \cdots, G_{i_{k}}^{t}\right\}, \quad 1 \leq k \leq n$, satisfy

$G_{i_{r}}^{t} \neq G_{i_{s}}^{t}, r \neq s$, that is, no repeated goals in

$G_{i}^{t}, 1 \leq i \leq n$. The algorithm for creating hierarchical goals is given as follows:

Algorithm 1: Hierarchical goals

Input: a set of goals $G_{i}^{t}=\left\{G_{i_{1}}^{t}, G_{i_{2}}^{t}, \cdots, G_{i_{k}}^{t}\right\}$ and their partial order

Output: $D A G$ which is a directed acyclic graph

1) Initialize $D A G=$ null; 
2) if $G_{i}^{t}$ is empty, then end and return $D A G$;

3) take $G_{i_{j}}^{t}$ from $G_{i}^{t}, 1 \leq j \leq k$, update $G_{i}^{t} \leftarrow G_{i}^{t}-\left\{G_{i_{j}}^{t}\right\}$;

4) if $G_{i}^{t}$ is empty, create root = new node ("root"), generate node $\left(G_{i_{j}}^{t}\right)$, and let parent $\left(\operatorname{node}\left(G_{i_{j}}^{t}\right)\right)=$ root ;

5) call insert $\left(\right.$ root, $\left.G_{i_{j}}^{t}\right)$;

6) Goto 2.

Function insert $\left(\right.$ root, $\left.G_{i_{j}}^{t}\right)$

1) let Children = children(root);

2) if Children is empty, create a new node $\operatorname{node}\left(G_{i_{j}}^{t}\right)$, and, parent $\left(\right.$ node $\left.\left(G_{i_{j}}^{t}\right)\right)=$ root , return;

3) take Child from Children, let

Children $\leftarrow$ Children $-\{$ Child $\}$;

4) if no partial order between Child. Concept and $G_{i_{j}}^{t}$, then Goto 2;

5) if Child .Concept $\prec G_{i_{j}}^{t}$, then create a new node $\operatorname{node}\left(G_{i_{j}}^{t}\right)$, let

$$
\begin{gathered}
\operatorname{parent}\left(\operatorname{node}\left(G_{i_{j}}^{t}\right)\right)=\operatorname{root}, \text { and } \\
\operatorname{parent}(\text { Child })=\operatorname{node}\left(G_{i_{j}}^{t}\right),
\end{gathered}
$$

delete parent $($ Child $)=$ root , return;

6) call insert (Child, $\left.G_{i_{j}}^{t}\right)$.

Comments:

1) Function children(node $C$ ) find all connected nodes directly with node $C$;

2) Function parent(node $C$ ) find directed parent node of node $C$;

3) Function new node $(C)$ create a new node $C$;

4) Function parent $(\operatorname{node}(C))=\operatorname{node}(D)$ assign node $D$ as a parent node to node $C$.

\subsection{Action Composition}

Action composition is the process of constructing a complex composite action from atomic actions to achieve a specific task. Action composition can be divided into two steps, first is action selection, i.e., select related action from action library. Then selected actions are composed together using a planning strategy.

The action selection chooses a single action from a just instantiated action stream or possibly from a previously active stream. There are a lot of selection methods. Most of them match goal and behavior based on similarity criteria.

Planning offers a scalable and efficient approach for action composition. It allows for a composition request to be expressed in terms of goal conditions that specify a set of constraints and preferences. We use dynamic description logic to describe definitions and algorithm for- mally [19].

Definition 2 (Action) An action description is the form of $A\left(x_{1}, \cdots, x_{n}\right) \equiv\left(P_{A}, E_{A}\right)$, where:

- $A$ is the action name.

- $x_{1}, \cdots, x_{n}$ are individual variables, which denote the objects on which the action operate.

- $P_{A}$ is the set of pre-conditions, which must be satisfied before the action is executed, i.e. $P_{A}=\{$ con $\mid$ con $\in$ condition .

- $E_{A}$ is the set of post-conditions, which denote the effects of the action, $E_{A}$ is a set of pair headbody, where head $=\{$ con $\mid$ con $\in$ condition $\}$, body is a condition.

Remark:

1) Action defines the transition relation of state, i.e. an action $A$ transit a state $u$ to a state $v$, if action $A$ can produce state $v$ under state $u$. The transition relation depends on whether state $u, v$ satisfy the pre-conditions and post-conditions of action $A$. The transition relation is denoted as $u T_{A} v$.

2) The definition of condition and action description reference [29,30].

Definition 3 (Parallel action stream) A parallel action stream $S$ is an action stream that is achieved by the independent relationship. $S=a_{1}\left|a_{2}\right| \cdots \mid a_{k}$, Which means the candidate actions in the stream $S$ can be independently executed.

Definition 4 (Sequence action stream) $S=a_{1} ; a_{2} ; \ldots$; $a_{k}$. A sequence service $S$ is a service that is achieved by the prerequisite relationship.

Definition 5 (Action stream composition problem) the composition problem can be described as a four-tuple < $T, A, G, S>$, where:

- $T$ describes the vocabulary of the application domain.

- A contains assertions about named individuals in terms of this vocabulary and also denotes the initial state of the world.

- $G$ is a set of assertions, which represent the goal attempting to reach.

- $S$ is the set of action stream as described before.

The algorithm for finding the appropriate action stream during the action composition is given in Algorithm 2.

Algorithm 2: Action composition

1) $T$ is the Tbox

2) $A$ is the ABox, add the initial state to $A$

3) $G$ is the Goal

4) $S=(S 1, \cdots, S n)$

5) GoalStreams $=\varnothing$

6) if $T, A \vDash G$ then

7) GoalStreams $=\varnothing$ return GoalStreams

8) end if

9) select an assertion subGoal from the unsatisfied goal $G$ 
10) restGoal $=G-$ subGoal

11 ) if the subGoal has already been processed by subStream then

12) executed recursively, and get restStreams to reach restGoal.

13) GoalStreams = subStreamsjrestStreams

14) return GoalStreams

15) else if $T, A \vDash$ subGoal then

16) executed recursively, and get restStreams to reach restGoal.

17) GoalStreams = restStreams

18) return GoalStreams

19) else if If no action stream satisfy the subgoal subGoal then

20) return $N U L L$

21) else

22) IF action stream $S^{\prime}=\left\{S^{\prime}{ }_{1}, \cdots, S^{\prime}{ }_{\mathrm{k}},\right\}$ satisfying the Subgoal

\section{3) loop}

24) select a set of streams subStreams from S' satisfying the subgoal

25) If a set of preconditions to satisfy the subgoal, $G^{\prime}=\{G 1, \cdots, G m\}$, where $G i=$ Pre(subStreams)+

\section{6) loop}

Posti(subStreams, subGoal)

27) select a preGoal from $G$ ' satisfying the subgoal

28) executed recursively, and get preStreams to reach preGoal.

29) executed recursively, and get restStreams to reach restGoal.

30) if preStreams $\neq N U L L$ then

31) if restStreams $\neq N U L L$ then

32) GoalStreams = (preStreams; subStreams) restStreams

33) return GoalStreams

34) end if

35) end if

36) remove preGoal from $G$,

37) end loop

38) remove subStreams from $S$,

39) end loop

40) return NULL

\section{1) end if}

Planning technologies differ in the complexity of the problems they can handle and the representations. We can employ different search algorithms to synthesis a plans and the constraints they observe. A number of different planning methodologies are developed, such as state space planning, plan space planning, planning graph techniques, hierarchical task network planning, model based planning. We will continue to consider which method is better for action composition in the mind model CAM.

\section{Discussion and Conclusions}

Cognitive cycle is a basic procedure of mental activities in cognitive level. Cognitive cycle should reflect agent interaction with environment, which is sensory outside environment, through deliberation, and then affect it. Traditional problem solving cycle can be embedded into cognitive cycle and looks like a particular situation to focus on solving questions. Here cognitive cycle is a whole procedure from perception to behavior execution.

Franklin and Baars [15] pointed out "A cognitive cycle can be thought of as a moment of cognition-a cognitive moment; higher-level cognitive processes are composed of many of these cognitive cycles, each a cognitive atom." And they proposed the cognitive cycle as "perceive-understand-act". This paper proposes the cognitive cycle in CAM, which is different with LIDA's cognitive cycle. We emphasize motivation and action composition. We use dynamic description logic to describe it formally.

We will continue to research on activities and algorithms for the each phase in cognitive cycle. The proposed cognitive cycle will be implemented by simulation in multi-agent systems.

\section{References}

[1] A. Newell, "Unified Theories of Cognition,” Harvard, Cambridge, 1990.

[2] J. Laird, A. Newell and P. Rosenbloom, "SOAR: An Architecture for General Intelligence,” Artificial Intelligence, Vol. 33, No. 1, 1987, pp. 1-64. doi:10.1016/0004-3702(87)90050-6

[3] J. R. Anderson, "The Adaptive Character of Thought," Erlbaum, Hillsdale, 1993.

[4] J. R. Anderson and C. Lebiere, "The Newell Test for a Theory of Cognition,” Behavioral and Brain Science, Vol. 26, No. 5, 2003, pp. 601-648. doi:10.1017/S0140525X0300013X

[5] J. R. Anderson and Y. Qin, "Using Brain Imaging to Extract the Structure of Complex Events at the Rational Time Band,” Journal of Cognitive Neuroscience, Vol. 20, No. 9, 2008, pp. 1624-1636. doi:10.1162/jocn.2008.20108

[6] J. R. Anderson, "Using Neural Imaging to Inform the Instruction of Mathematics,” In: N. Zhong, K. C. Li, S. F. $\mathrm{Lu}$ and L. Chen, Eds., Brain Informatics, International Conference, 2009 International Conference on Brain Informatics, Beijing, 23-24 October, 2009, p. 1.

[7] J. R. Anderson, S. A. Betts, J. L. Ferris and J. M. Fincham, "Neural Imaging to Track Mental States While Using an Intelligent Tutoring System," Proceedings of the National Academy of Science, Vol. 107, No. 15, 2010, 
pp. 7018-7023._doi:10.1073/pnas.1000942107

[8] Z. Z. Shi, “Advanced Artificial Intelligence,” World Scientific Publishers, Hackensack, 2011.

[9] Y. Shoham, “Agent-Oriented Programming," Artificial Intelligence, Vol. 60, No. 1, 1993, pp. 51-92. doi:10.1016/0004-3702(93)90034-9

[10] S. Yoav and K. Leyton-Brown, "Multiagent Systems: Algorithmic, Game-Theoretic, and Logical Foundations," Cambridge University Press, Cambridge, 2008.

[11] M. Georgeff, B. Pell, M. Pollack, M. Tambe and M. Wooldridge, "The Belief-Desire-Intention Model of Agency," Intelligent Agents V. Agent Theories, Architectures, and Languages, 5th International Workshop, ATAL’98, Paris, 4-7 July 1998, pp. 630-630.

[12] R. Sun and X. Zhang, "Top-Down versus Bottom-up Learning in Cognitive Skill Acquisition,” Cognitive Systems Research, Vol. 5, No. 1, 2004, pp. 63-69. doi:10.1016/j.cogsys.2003.07.001

[13] R. Sun, "Motivational Representations within a Computational Cognitive Architecture,” 2008. http://www.cogsci.rpi.edu/ rsun/folder-files/sun-c

[14] S. Franklin, B. J. Baars, U. Ramamurthy and M. Ventura, "The Role of Consciousness in Memory," Brains, Minds and Media, No. 1, 2005, pp. 1-38.

[15] B. J. Baars and S. Franklin, "Consciousness Is Computational: The LIDA Model of Global Workspace Theory,” International Journal of Machine Consciousnesss, Vol. 1, No. 1, 2009, pp. 23-32. doi:10.1142/S1793843009000050

[16] T. Madl, B. J. Baars and S. Franklin, "The Timing of the Cognitive Cycle,” Plos One, Vol. 6, No. 4, 2011, Article ID e14803. doi:10.1371/journal.pone.0014803

[17] Medical Dictionary Website, 2003. http://www.medterms.com/script/main/hp.asp

[18] Z. Z. Shi and X. F. Wang "A Mind Model CAM in Intelligence Science,” International Journal of Advanced Intelligence, Vol. 3, No. 1, 2011, pp. 119-129.

[19] Z. Z. Shi, M. K. Dong, Y. C. Jiang and H. J. Zhang, “A Logic Foundation for the Semantic Web," Science in China, Series F Information Sciences, Vol. 48, No. 2, 2005, pp. 161-178. doi:10.1360/03yf0506
[20] A. M. Collins and M. R. Quillian, "Retrieval Time from Semantic Memory," Journal of Verbal Learning and Verbal Behavior, Vol. 8, No. 2, 1969, pp. 240-247. doi:10.1016/S0022-5371(69)80069-1

[21] E. Tulving, "Elements of Episodic Memory,” Oxford Clarendon Press, London, 1983.

[22] A. Nuxoll and J. E. Larid, "Extending Cognitive Architecture with Episodic Memory," Proceedings of the $22^{\text {nd }}$ AAAI Conference on Artificial Intelligence, Vol. 2, 2007, pp. 1560-1564.

[23] G. Tononi, G. M. Edelman and O. Sporns, "Complexity and Coherency: Integrating Information in the Brain," Trends in Cognitive Sciences, Vol. 2, No. 12, 1998, pp. 474-484. doi:10.1016/S1364-6613(98)01259-5

[24] P. Langley, J. E. Laird and S. Rogers, "Cognitive Architectures: Research Issues and Challenges,” Cognitive Systems Research, Vol. 10, No. 2, 2009, pp. 141-160. doi:10.1016/j.cogsys.2006.07.004

[25] A. H. Maslow, “A Theory of Human Motivation,” Psychological Review, Vol. 50, No. 4, 1943, pp. 370-396. doi: $10.1037 / \mathrm{h} 0054346$

[26] A. H. Maslow, R. Frager and J. Fadiman, "Motivation and Personality,” 3rd Edition, Addison-Wesley, Boston, 1987.

[27] J. Bach, "A Motivational System for Cognitive AI," AGI'11 Proceedings of the 4th International Conference on Artificial General Intelligence, LNAI 6830, 2011, pp. 232-242.

[28] J. Bach, "PSI-An Architecture of Motivated Cognition," Oxford University Press, Oxford, 2009.

[29] F. Baader, M. Milicic, C. Lutz, U. Sattler and F. Wolter, "Integrating Description Logics and Action Formalisms for Reasoning about Web Services,” LTCS-Report LTCS-05-02, Chair for Automata Theory, Institute for Theoretical Computer Science, Dresden University of Technology, Dresden, 2005. http://lat.inf.tudresden.de/research/reports.html

[30] C. Lutz and U. Sattler, "A Proposal for Describing Services with DLs,” In: I. Horrocks and S. Tessaris, Eds., Proceedings of the 2002 International Workshop on Description Logics, Toulouse, 19-21 April 2002, pp. 129140. 